\title{
THE GAUSS MAP IN SPACES OF CONSTANT CURVATURE
}

\author{
JOEL L. WEINER
}

\begin{abstract}
Let $N$ be a complete simply connected Riemannian manifold of constant sectional curvature $\neq 0$. Let $M$ be an immersed Riemannian hypersurface of $N$. The Gauss map on $M$ based at a point $p$ in $N$ is defined. Suppose a Gauss map on $M$ has constant rank less than the dimension of $M$; then $M$ is generated by Riemannian submanifolds with constant sectional curvature. The sectional curvature of each of these generating submanifolds of $M$ has the same sign as the sectional curvature of $N$.
\end{abstract}

1. Introduction. Let $M$ be an $n$-dimensional Riemannian manifold isometrically immersed into Euclidean $(n+k)$-space $E^{n+k}(k \geqq 1)$ and $v$ be the bundle of unit vectors normal to $M$. The Gauss map of $v$ into the unit sphere $S^{n+k-1}$ about the origin of $E^{n+k}$ is well known. Willmore and Saleemi [3] generalized this map to the case where $M$ is an $n$-dimensional Riemannian manifold immersed into an $(n+k)$-dimensional complete, simply connected Riemannian manifold $N$ with nonpositive sectional curvature as follows. Let $p \in N$ and $v$ be the unit normal bundle of $M$ in $N$. The parallel displacement of $v \in v$ along the shortest geodesic joining the foot point of $v$ to $p$ gives a mapping of $v$ into the unit sphere in the tangent space of $N$ at $p$. If $N$ is an arbitrary Riemannian manifold with $M$ isometrically immersed into it and $p \in N$ the only requirement we need for the construction of Willmore and Saleemi is for $M$ not to intersect the cut locus of $p$. We call the resulting mapping from $v$ into the unit sphere in the tangent space of $N$ at $p$ the Gauss map on $M$ based at $p$.

$\mathrm{R}$. Takagi [2] describes an $n$-dimensional complete Riemannian manifold $M$ isometrically immersed into an Euclidean $(n+1)$-sphere $S^{n+1}$ when the Gauss map on $M$ based at a point $p \in S^{n+1}$ has constant rank on $M$. He shows that $M$ is generated by metric spheres. We will show that a similar result holds when the ambient space is a complete, simply connected Riemannian manifold of constant sectional curvature -1 , that is, hyperbolic space of curvature -1 . In this case, $M$ is generated by hyperbolic spaces. We will also reprove Takagi's theorem in a simpler fashion.

Received by the editors May 10, 1972 .

AMS (MOS) subject classifications (1970). Primary 53C40; Secondary 53A30.

Key words and phrases. Parallel displacement, Gauss map, submanifold of a sphere, submanifold of hyperbolic space, conformally equivalent, constant rank, foliated, constant sectional curvature.

(c) American Mathematical Society 1973 
Definition. We say a Riemannian manifold is generated by Riemannian manifolds of a certain type if we can foliate $M$ into Riemannian submanifolds of that type.

2. The Gauss map in $H^{n}$. Let $H^{n}$ be $n$-dimensional hyperbolic space of constant sectional curvature $-1 . H^{n}$ will be realized as $\left\{x \in E^{n}:\|x\|<1\right\}$ with the metric $\langle$,$\rangle where \langle v, w\rangle=(v \cdot w) / h^{2}(x)$ if $v$ and $w$ are vectors at $x$ and $h(x)=\left(1-\|x\|^{2}\right) / 2$. Thus the metric on $H^{n}$ is conformally equivalent to the usual flat metric on $\left\{x \in E^{n}:\|x\|_{i}<1\right\}$.

Suppose $M$ is an $n$-dimensional Riemannian manifold isometrically immersed in $H^{n+k}(k \geqq 1)$. Let $\nu_{H}(M)$ be the unit normal bundle of $M$ in $H^{n+k}$. Let $p \in M$ and $e_{H}: v_{H}(M) \rightarrow S_{p} H^{n+k}$ be the Gauss map on $M$ based at $p$, where $S_{p} H^{n+k}$ is the unit sphere in tangent space to $H^{n+k}$ at $p$. If $p=0$, the origin in $E^{n+k}$, we identify $H_{p}^{n+k}=H_{0}^{n+k}$ with $E^{n+k}$; then $S_{0} H^{n+k}=$ $S^{n+k-1}(1 / 2)$, the sphere about the origin in $E^{n+k}$ of radius $1 / 2$. The radius is $1 / 2$ because $h(0)=1 / 2$; that is, the metric on $H_{0}^{n+k}$ is twice the usual metric on $E^{n+k}$.

$M$ is also immersed in $E^{n+k}$ (of course, not isometrically). Let $v_{E}(M)$ be the unit normal bundle of $M$ in $E^{n+k}$. Let $e_{E}: v_{E}(M) \rightarrow S^{n+k-1}(1)$ be the usual Gauss map in $E^{n+k}$.

LEMMA 1. Let $M$ be an n-dimensional Riemannian manifold isometrically immersed in $H^{n+k}$. Let $e_{H}$ be the Gauss map based at 0 . Then the following diagram is commutative, where $h(v)=h(x) v$ if $v \in v_{E}(M)_{x}$, that is, $v$ is normal to $M$ at $x$, and $\times 1 / 2$ indicates scalar multiplication by $1 / 2$.

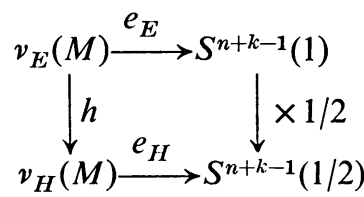

Proof. Let $v \in v_{E}(M)_{x}$; then $h(x) v \in v_{H}(M)_{x}$. Let $\zeta:[0, \rho] \rightarrow H^{n+k}$ be the unit speed geodesic from $x \in M$ to 0 . We know that $\zeta$ is a reparametrization of the straight line segment in $E^{n+k}$ from $x$ to 0 . Let $V$ be the parallel vector field along $\zeta$ in $E^{n+k}$ such that $V(0)=v$. Then $(h \circ \zeta) V$ is the parallel vector field along $\zeta$ in $H^{n+k}$ such that $[(h \circ \zeta) V](0)=h(x) v$. Hence, $e_{H}(h(x) v)=h \circ \zeta(\rho) V(\rho)=(1 / 2) V(\rho)=(1 / 2) e_{E}(v)$.

LEMMA 2. $\quad h: v_{E}(M) \rightarrow v_{H}(M)$ is a diffeomorphism, and hence the rank of $e_{E}$ at $v$ equals the rank of $e_{H}$ (based at 0 ) at $h(v)$ for all $v \in \nu_{H}(M)$.

Proof. Both statements are obvious.

REMARK. Lemmas 1 and 2 essentially still hold if $H^{n+k}$ is replaced by a Riemannian manifold $N^{n+k}$ defined on the open unit disk in $E^{n+k}$ with 
metric $\langle$,$\rangle , where \langle v, w\rangle=(v \cdot w) / h^{2}(x)$, if $v$ and $w$ are vectors at $x$, and $h(x)$ is a positive function which depends only on $\|x\|$.

3. Special hyperbolic subspaces. Before we proceed further it is necessary to characterize intrinsically the Riemannian submanifolds $M=L^{n} \cap$ $H^{n+1}$ of $H^{n+1}$, where $L^{n}$ is a hyperplane of $E^{n+1}$ which has nontrivial intersection with $H^{n+1}$.

Let $d$ be the metric on $H^{n+1}$. Fix $p \in H^{n+1}$ and let $M$ be an $n$-dimensional complete orientable Riemannian submanifold. Set $\lambda=\tanh (d(p, M) / 2)$. Let $z \in M$ such that $d(p, z)=d(p, M)$. Let $U$ be a unit normal vector field on $M$ such that $U(z)=\dot{\zeta}(0)$, the initial velocity of the unit speed geodesic $\zeta$ from $z$ to $p$, if $z \neq p$. If the second fundamental form $S_{U}$ with respect to $U$ equals $\lambda I$, where $I$ is the identity, then $M$ is called a special hyperbolic subspace with respect to $p$. Indeed, for $n \geqq 2, M$ is a hyperbolic space with constant sectional curvature $-1+\lambda^{2}<0$. If $\lambda \neq 0, M$ is one of two totally umbilic hyperbolic spaces through $z$ at a distance $d(p, z)$ from $p$; for the other space $S_{U}=-\lambda I$. If $\lambda=0, M$ is a totally geodesic hypersurface through $p$.

Isometries preserve the relationship of being a special hyperbolic subspace with respect to a point. If $\phi: H^{n+1} \rightarrow H^{n+1}$ is an isometry, $p \in H^{n+1}$, and $M$ is a special hyperbolic subspace with respect to $p$, then $\phi(M)$ is a special hyperbolic subspace with respect to $\phi(p)$. Hence if we know the special hyperbolic subspaces with respect to one point of $H^{n+1}$, then we know the special hyperbolic subspaces with respect to any point.

LEMMA 3. The special hyperbolic subspaces of $H^{n+1}$ with respect to 0 are the Riemannian submanifolds $L^{n} \cap H^{n+1}$, where $L^{n}$ is a hyperplane of $E^{n+1}$ which has nontrivial intersection with $H^{n+1}$.

Proof. The calculations are straightforward. They depend heavily on the fact that $H^{n+1}$ has a metric which is conformally equivalent to the usual flat metric on the open unit disk by a function which depends only on $\|x\|$.

4. Theorem. If $M$ is orientable afd $k=1$, we can identify $M$ with a component of $v_{H}(M)$ and also the corresponding component of $v_{E}(M)$. Then $e_{H}: M \rightarrow S_{p} H^{n+1}$ is the Gauss map based at $p$ and $e_{E}: M \rightarrow S^{n}(1)$ is the usual Gauss map.

THEOREM 4. Let $M$ be an n-dimensional complete orientable Riemannian manifold isometrically immersed in $H^{n+1}$. Suppose $e_{H}: M \rightarrow S_{p} H^{n+1}$ has constant rank $n-m$ on $M(0 \leqq m \leqq n)$.

(1) Let $m=0$. If $M$ is compact, then $M$ is diffeomorphic to the $n$-sphere. 
(2) Let $1 \leqq m \leqq n-1$. Then $M$ is generated by m-dimensional hyperbolic spaces (whose sectional curvatures may vary).

(3) Let $m=n$. Then $M$ is a special hyperbolic subspace with respect to $p$, and conversely.

Proof. (1). This is clear.

(2) and (3). First, we may assume without loss of generality that $p=0$ by the homogeneity of $H^{n+1}$. Thus $e_{H}: M \rightarrow S^{n}(1 / 2)$ having constant rank $n-m$ on $M$ implies that $e_{E}: M \rightarrow S^{n}(1)$ has constant rank $n-m$ on $M$, by Lemma 2. By Lemma 2 of [1], $M$ is generated by $m$-dimensional planes $L^{m}$ in $E^{n+1}$ intersected with $H^{n+1}$. For each $L^{m}, L^{m} \cap H^{n+1}$ with the metric induced from $H^{n+1}$ is a hyperbolic space. Since the metric on $L^{m} \cap H^{n+1}$ depends on the distance from $L^{m}$ to 0 , the curvatures of these hyperbolic spaces may vary. Hence $M$ is generated by $m$-dimensional hyperbolic spaces.

When $m=n, M$ is a hyperplane $L^{n}$ intersected with $H^{n+1}$ with the induced metric. Thus, by Lemma 3 , it is a special hyperbolic space with respect to 0 . If, on the other hand, $M$ is a special hyperbolic space with respect to 0 , then there exists a hyperplane $L^{n}$ of $E^{n+1}$ such that $M=L^{n} \cap H^{n+1}$. Hence $e_{E}: M \rightarrow S^{n}(1)$ has rank 0 ; hence, $e_{H}: M \rightarrow S_{0} H^{n+1}$ has rank 0 .

The preceding theorem is also true on hyperbolic spaces with constant sectional curvature different from -1 . However, it is necessary to modify the definition of special hyperbolic subspace so that $S_{U}=(K \lambda) I$ on a special hyperbolic subspace when the ambient hyperbolic space has constant sectional curvature $-K<0$.

5. The Gauss map in spheres. Let $S^{n+1}$ be the Euclidean unit $(n+1)$ sphere. Fix $p \in S^{n+1}$. Suppose $M$ is an $n$-dimensional orientable immersed submanifold of $S^{n+1}$, and $v_{S}(M)$ is the unit normal bundle of $M$ in $S^{n+1}$. Since the codimension of $M$ in $S^{n+1}$ is 1 and $M$ is orientable, we identify $M$ with a component of $v_{S}(M)$.

Let $-p$ denote the antipode of $p$. Since $-p$ is the cut locus of $p$ we can define the Gauss map based at $p$ as $e_{S}: M \backslash\{-p\} \rightarrow S_{p} S^{n+1}$.

Let $\sigma: S^{n+1} \mid\{-p\} \rightarrow E^{n+1}$ be stereographic projection from $-p ; \sigma$ is a conformal mapping. Denote by $M^{*}$ the image of $M \backslash\{-p\}$ under $\sigma . \sigma(p)=0$, so we can identify the tangent space at $p$ with $E^{n+1}$. Under this identification we know that $S_{p} S^{n+1}$, the unit sphere about $p$ in the tangent space to $S^{n+1}$ at $p$, agrees with $S^{n}$, the unit sphere about 0 in $E^{n+1}$. Hence $e_{S}$ : $M \backslash\{-p\} \rightarrow S^{n}$. Let $e_{E}: M^{*} \rightarrow S^{n}$ be the usual Gauss map in $E^{n+1}$ on $M^{*}$.

Lemma 5. Let $M$ be an n-dimensional orientable Riemannian manifold isometrically immersed in $S^{n+1}$. Then the following diagram is commutative. 


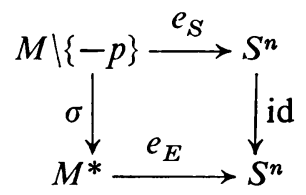

Proof. The proof is identical to the proof of Lemma 1.

Using Lemma 5, the fact that planes in $E^{n+1}$ correspond to spheres through $-p$ under $\sigma$, and arguing as in the hyperbolic case we get the theorem first due to R. Takagi [2], which we restate here in an intrinsic form.

THEOREM 6. Let $M$ be an $n$-dimensional complete orientable Riemannian manifold isometrically immersed in $S^{n+1}$. Suppose that the Gauss map $e_{S}: M \backslash\{-p\} \rightarrow S_{p} S^{n+1}$ based at $p$ has constant rank $n-m$ on $M \backslash\{-p\}$ $(0 \leqq m \leqq n)$.

(1) Let $m=0$. If $M$ is compact, then $M$ is diffeomorphic to the $n$-sphere.

(2) Let $1 \leqq m \leqq n-1$. Then $M$ is generated by Euclidean $m$-spheres, each through $-p$.

(3) Let $m=n$. Then $M$ is a Euclidean hypersphere through $-p$, and conversely.

Again, we remark that the preceding result is not peculiar to Euclidean spheres of unit radius but holds for Euclidean spheres of arbitrary radius.

REMARK. It is important to note that the last two parts of Theorem 4 and Theorem 6 are really local in nature. Thus, if the Gauss map based at $p$ on an open set $V \subset M$ has constant rank less than $n$, then the open set $V$ is generated by parts of special hyperbolic subspaces with respect to $p$ or parts of Euclidean spheres through $-p$ according as the ambient space has constant negative or positive sectional curvature.

\section{REFERENCES}

1. S. S. Chern and R. K. Lashof, On the total curvature of immersed manifolds, Amer. J. Math. 79 (1957), 306-313. MR 18, 927.

2. R. Takagi, Gauss map in a sphere, Kōdai Math. Sem. Rep. 22 (1970), 82-88.

3. T. J. Willmore and B. A. Saleemi, The total absolute curvature of immersed manifolds, J. London Math. Soc. 41 (1966), 153-160. MR 32 \#3019.

Department of Mathematics, University of California, los Angeles, CaliFORNIA 90024

Current address: Department of Mathematics, Michigan State University, East Lansing, Michigan 48823. 